\title{
STUDIES ON THE VEGETATIVE PROPAGATION OF Gymnema sylvestry (MASBEDDA)
}

\author{
DLC Kumari, KKIU Aruna Kumara, GA Dayatilake and R Senarathne \\ Department of Crop Science, Faculty of Agriculture, University of Ruhuna, Mapalana
}

Gymnema is a woody, extensively branched perennial yine well known as a medicinal plant with anti-diabetic properties. The extract of leaves contains gymnemic acid and a mixture of triterpene-glycosides known as gymnemosides, which were found effective in reducing blood sugar by acting indirectly through the stimulation of insulin secretion of the pancreas. Further, it was found that, it reduces the serum cholesterol levels, stimulate circulatory system and also used in treating snakebites. Thus, Gymnema has a ready demand in local and foreign market of herbal products, causing over exploitation of natural habitats, at the face of the lack of systemic cultivation. In this background, the current study was carried out to develop a cost effective protocol for the rapid multiplication of Gymnema.

It was a challenging task to find out sufficient amount of planting material, since Gymnema is a very rare species of plant. After an extensive survey, few authentic mother vines were identified from two locations in Matara district and one location in Monaragala district. The $1^{\text {st }}$ and the immediate phase of propagation studies were conducted on vegetative propagation, since the collection of seeds was extremely difficult. Three types of stem cuttings (2-3 nodal cuttings with leaves) i.e.; softwood (green, terminal portions), semi-hardwood (middle portion) and hardwood (basal portion) were used with hormone treatments and different potting mixtures. Application of Secto (NAA $0.3 \%$ + Thiram $0.2 \%$ in WP form) resulted $80 \%$ success in root formation followed by $50 \%$ success with the application of Clonex (3-IBA, $3 \mathrm{mg} / \mathrm{L}$ in paste form) compared to $10 \%$ success observed in the control

It could be clearly observed from the results that, Gynnema can be vegetatively propagated by the use of stem cuttings of specially the semi-hardwood type. Auxin based commercial root inducing hormone mixtures can be effectively utilizer to improve the success of root formation in Gymnema, A potting medium with desirable properties i.e. high moisture holding capacity while maintaining good aeration can further enhance the success of vegetative propagation. Thus, it can be concluded from the results that, the mass propagation of Gymnema can be achieved within a reasonably short period of time (less than one year) by using the technique developed. 